\title{
RESEARCH AND GOOGLE TREND FOR HUMAN NEUROPSYCHIATRIC DISORDERS AND MACHINE LEARNING: A BRIEF REPORT
}

\author{
Manik Sharma \\ Department of CSA, DAV University Jalandhar, Jalandhar, India
}

received: 25.4 .2021 ;

revised: 5.6.2021;

accepted: 10.6.2021

\section{SUMMARY}

Objectives: The key characteristics of this study are to highlight the research trend pertaining to the use of machine learning in the diagnosis and management of neuropsychiatric conditions.

Methods: The last ten years (2011-2020) Scopus data related to the use of machine learning techniques in the diagnosis and management of neuropsychiatric disorders in human beings have been collected and examined using VOSviewer. The global internet trend for neuropsychiatric disorders and machine learning techniques during the observation period (1-Jan-2010 to 30-Nov-2020) has been also explored using Google Trend.

Results: The mean values of the Google trend for neuropsychiatric disorders and machine learning are 52.09 and 40.00 respectively. Moreover, the correlation coefficient for the Google trend of USA, UK and the world found to be significantly (0.98) higher. Likewise, the mean values of web trend for USA, UK, and China are 42.17, 38.55, and 30.90. Additionally, the Google trend for the term 'machine learning' in the observation period (1-Jan-2010 to 30-Nov-2020) has been also explored.

Conclusion: It is observed that the researchers from the US (32.4\%), UK (9.2\%) and China (7.4\%) are the prime contributors as far as mining and management of the neuropsychiatric disorders using machine learning is concerned. Moreover, the study revealed that neuropsychiatric disorders (seizure, eating, mood, sleep, conduct, and intellectual) need more attention as far as machine learning is concerned.

Key words: neuropsychiatric disorders - machine learning - google trend

$* * * * *$

\section{INTRODUCTION}

Neuropsychiatric disorders entail a broad range of medical conditions that span both neurology and psychiatry (Gautam \& Sharma 2020, Pacheco-Lorenzo et al. 2020). Seizure, anxiety, depression, palsies, migraine, addiction, ADHD, are some of the key neuropsychiatric issues. In general, infection, environmental factors, traumatic head injuries, medication side effects, are the key inducers of these brain disorders. These mental disorders can make a person physically or mentally disabled (Wichmann \& DeLong 2006). These disorders may affect the sleep, attention, eating, mood, conduct, behaviour and intellectual level of the victims. Children, adults and old aged people are equally affected by these human disorders. Autism, pervasive development, and attention deficit hyperactivity disorders are common childhood related neuropsychiatric disorders. Likewise, Alzheimer, Parkinson, and stroke are common neuropsychiatric conditions among old aged persons.

The early diagnosis of these neuropsychiatric conditions can save masses of human lives. In the last two decades, several computing techniques have been used to diagnose different human brain disorders.

Machine Learning (ML) techniques are artificial intelligent methods that assist in automatic recognition (without explicit programming) of dimension, position, pattern, and deep interpretation of the data. In general, the ML techniques synthesize significant facts and concepts to solve distinct real-life classification, clustering, regression and predictive problems (Jordan \& Mitchell 2015, Bose \& Mahapatra 2011). Recently, distinct ML techniques have been used in the diagnosis of some of the different psychiatric, neuropsychiatric, and neurological human disorders (Kaur \& Sharma 2019).

The key characteristics of this study are to highlight the research trend pertaining to the use of ML in the diagnosis and management of neuropsychiatric conditions. The Google trend waves for ML techniques have also been highlighted for United States (USA), United Kingdom (UK), and China. The data for this study has been gleaned from the Scopus indexing database and Google trends (Google Trends).

\section{Related Works}

Earlier, different studies have been carried out to explore the publishing and research trend for disaster health (Kelen \& Sauer 2008), public health research (Chuang et al. 2011), open access (Poltronieri et al. 2016), clinical radiology (Rahman et al. 2005), predatory journals (Lukić et al. 2014), epidemiology (Takahashi et al. 1996), health services (Wisdom et al. 2012), ergonomics (Lee 2010), feature selection (Sharma \& Kaur, 2020 ), and mariner health (Sharma 2021). However, a little attention is given to mine the research trend of neuropsychiatric disorders using machine learning. 


\section{METHODS}

The research trend of the Scopus indexing data (last ten years) related to neuropsychiatric disorders and their diagnosis using machine learning techniques has been investigated. A suitable query has been designed and executed to get and examine the data. Here, the results lie those between January 1, 2010, to November 30, 2020, have been mined. The results obtained through the Scopus database have been further analyzed using a bibliometric tool (VOSViewer).

\section{RESULTS AND DISCUSSIONS}

Neuropsychiatry diseases are mental disorders that affects the learning and working capacity of the individuals. It encompasses wide sphere of neurology and psychiatric medical conditions. Based on the condition, it may induce anger, depression, mood, eating and sleep disorder. It may also trigger attention, cognitive and perceptual deficits, which may ultimately affect the memory, attention and reasoning capability of the victims (Hyman 2008).

Based on the aforementioned query, 73 articles related to the use of ML techniques in the diagnosis and management of neuropsychiatric conditions have been identified. As per Scopus records, 1035 distinct keywords have been acknowledged for these articles. For a minimum of two occurrences of the keywords, out of 1035 distinct keywords, only 274 meets the threshold. The co-occurrence map for the same is presented in Figure 1. Based upon the total link strength of keyword occurrence network map, Figure 1 witnessed that the ML techniques have been used in mining magnetic resonance images, autism, parkinson, alzheimer, depression, and schizophrenia. The use of a support vector machine, feature learning, k-means clustering, and artificial neural network in mining and management of neuropsychiatric disorders has also been acknowledged. Additionally, the classification of mental diseases and brain mapping have also been explored using ML techniques. The direct or indirect use of ML in analysis of disease classification, genetic association, computerassisted diagnosis, biomarker, extracting clinical features, spectrum analysis, gene mutation, auditory situation, physiologic monitoring, and medical imaging has been recognized. However, no direct keyword connection of machine learning for mining of seizure, eating, mood, sleep, conduct, and intellectual disorders has been found. Therefore, more attention is needed in these areas.

The year-wise distribution of the articles published during 2010-2020 has been examined. A positive publication growth rate has been observed. The rate of article indexed in last ten years is $2010(1 \%), 2012$ (1\%), 2013 (6\%), 2014 (8\%), 2015 (7\%), 2016 (11\%), $2017(7 \%), 2018(10 \%), 2019(22 \%)$, and $2020(27 \%)$.

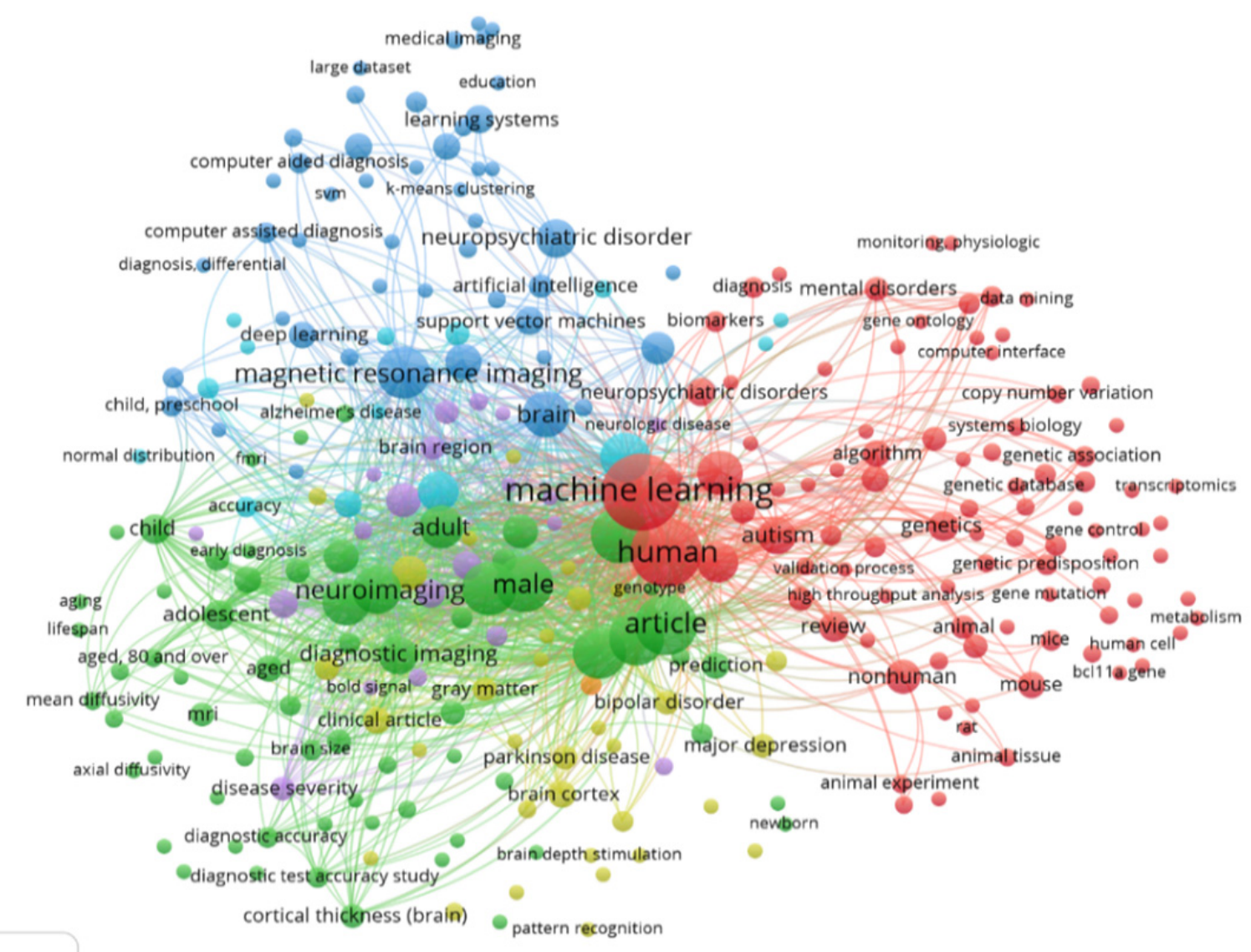

is VOSviewer

Figure 1. Keyword Co-occurrence Map 

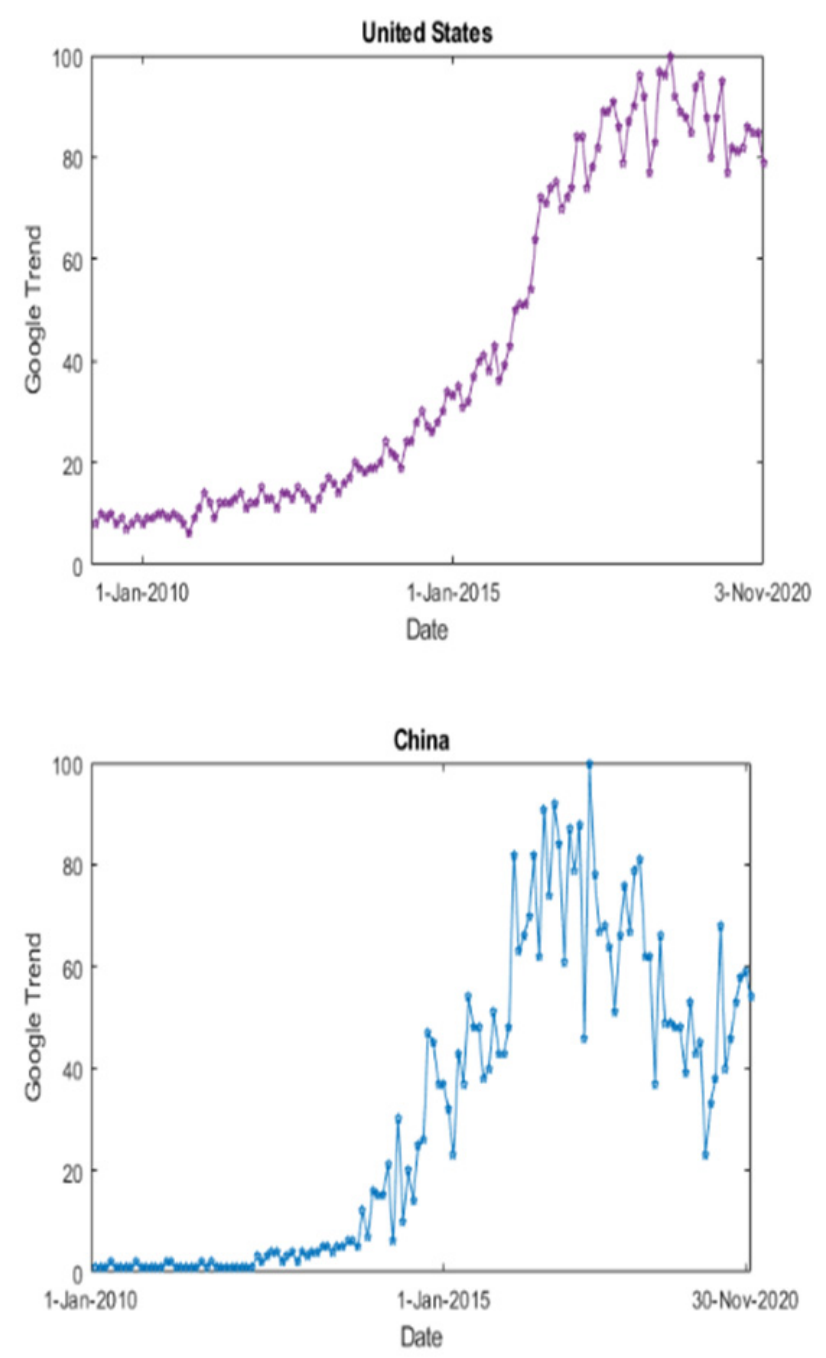

Figure 2. Google Trend (Machine Learning)

Amazingly, 49\% of these articles have been published in the last two years. The country-wise contribution of the articles (mining and management of neuropsychiatric disorders using machine learning) along with their cumulative citation has also been extracted. Surprisingly, only 32 distinct countries researchers are working in the area of mining and management of neuropsychiatric disorders using machine learning. It is observed that the researchers from the USA $(32.4 \%)$, UK $(9.2 \%)$ and China $(7.4 \%)$ are the prime contributors to these articles. Whereas, more effort is required from countries viz. India, Iran, Israel, Malaysia, Poland, Sweden, Saudi Arabia, Portugal and Thailand. The credit of top citations goes to the USA, UK, and China. However, if the average number of citations per document is recognized then India, Israel and Ireland are on the top list.

Moreover, the ratio of open-access and subscription-based articles is $36.9 \%$ (27), and 63.1 (46) respectively. Based on category, the percentage of articles, conference papers, reviews, book chapters, books, data papers, and short surveys is $64.3 \%, 13.6 \%, 12.3 \%$, $5.4 \%, 1.36 \%, 1.36 \%$, and $1.36 \%$ respectively.
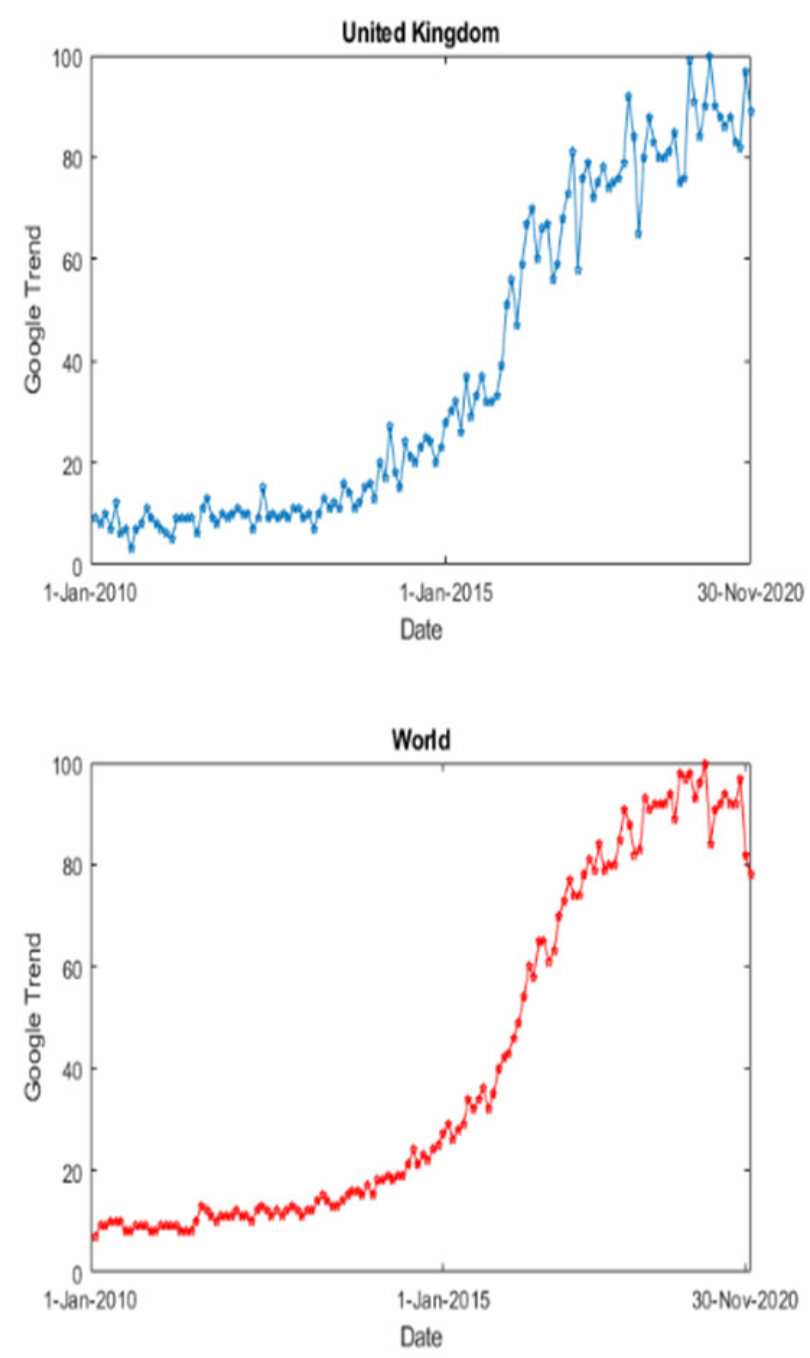

The global Google trend for neuropsychiatric disorders and machine learning techniques during the observation period (1-Jan-2010 to $30-N o v-2020$ ) has been also explored. The mean values of the Google trend for neuropsychiatric disorders and machine learning are 52.09 and 40.00 respectively. The Google trend for ML of the United States, United Kingdom, China and the world has been accessed. Figure 2 witnessed a positive web trend for ML. The correlation of Google trend of USA, UK, China and the world is $0.98,0.98$, and 0.81 respectively. Moreover, the mean values of web trend for USA, UK, and China are $42.17,38.55$, and 30.90. The p-values for the Google trend of USA and world, UK and world, and China and world are $0.633,0.676$, and 0.016 respectively, which proves that there is significant variation in the population means of these values.

\section{CONCLUSION}

In general, a total of 7899 articles related to neuropsychiatric disorders have been published in the last ten years (2011-2020). However, only 73 articles witnessed 
the use of ML in the diagnosis and management of neuropsychiatric disorders. No doubt, the utilization of $\mathrm{ML}$ in the diagnosis and management of neuropsychiatric conditions is growing. However, there is indeed a lot of work to be carried out. Some of the neuropsychiatric disorders (seizure, eating, mood, sleep, conduct, and intellectual) still need to be effectively mined using distinct machine learning techniques. The use of natural language processing techniques viz. keyword extraction, summarization will further help in identifying the areas of neuropsychiatric disorders for which the use of ML techniques has still need to be mined. A decent average rate of google trend for neuropsychiatric disorders and machine learning has been observed. A strong correlation coefficient for the Google trend of USA, UK and the overall world has been witnessed. In future, the semantic analysis of these articles can be done and used to get additional and more valuable insights.

\section{Acknowledgements: None.}

\section{Conflict of interest: None to declare.}

\section{References}

1. Bose I \& Mahapatra RK: Business data mining - a machine learning perspective. Information and Management 2011; 39:211-225

2. Chuang KY, Chuang YC, Ho $M \& H o$ YS: Bibliometric analysis of public health research in Africa: The overall trend and regional comparisons. South African Journal of Science 2011; 107:54-59

3. Gautam $R \&$ Sharma M: Prevalence and Diagnosis of Neurological Disorders Using Different Deep Learning Techniques: A Meta-Analysis. Journal of Medical Systems 2020; 44: 1-24

4. Google Trends. https://trends.google.com/trends/explore? date $=2010-01-01 \% 202020-11-30 \& q=\% 2 \mathrm{Fm} \% 2 \mathrm{~F} 01 \mathrm{hyh}$ (Accessed on $1^{\text {st }}$ Dec 2020)

5. Jordan MI \& Mitchell TM: Machine learning: Trends, perspectives, and prospects. Science 2015; 349:255-260

6. Hyman SE: A glimmer of light for neuropsychiatric disorders. Nature 2008; 455:890-893
7. Kaur P, Sharma M: Diagnosis of human psychological disorders using supervised learning and nature-inspired computing techniques: a meta-analysis. Journal of medical systems 2019; 43:1-30

8. Kelen GM \& Sauer LM: Trend analysis of disaster health articles in peer-reviewed publications pre-and-post-9/11. American journal of disaster medicine 2008; 3: 369-376.

9. Lee DH: A Study on Trend of the Research Papers Published in the Journal of the Ergonomics Society of Korea. Journal of the Ergonomics Society of Korea 2010; 29:701-707

10. Lukić T, Blešić, I, Basarin B, Ivanović-Bibić L, Milošević, $D \&$ Sakulski, D: Predatory and fake scientific journals/ publishers: A global outbreak with rising trend: A review. Geographica Pannonica 2014; 18:69-81

11. Pacheco-Lorenzo MR, Valladares-Rodríguez SM, AnidoRifón LE \& Fernández-Iglesias MJ: Smart onversational agents for the detection of neuropsychiatric disorders: A systematic review. Journal of Biomedical Informatics 2020; 103632

12. Poltronieri E, Bravo E, Curti M, Ferri $M \&$ Mancini $C$ : Open access publishing trend analysis: statistics beyond the perception. Information Research 2016; 21

13. Rahman M, Haque TL \& Fukui T: Research Articles Published in Clinical Radiology Journals: Trend of Contribution from Different Countries. Academic Radiology 2005; 12:825-829

14. Sharma $M \&$ Kaur P: A Comprehensive Analysis of Nature-Inspired Meta-Heuristic Techniques for Feature Selection Problem. Archives of Computational Methods in Engineering 2021; 28:1103: 1127

15. Sharma M: Visualisation and bibliometric analysis of worldwide research trend of stress among seafarer: an extensive publication analysis. International Maritime Health 20201; 72:64-75

16. Takahashi K, Hoshuyama T, Ikegami K, Itoh T, Higashi $T$ \& Okubo T: A bibliometric study of the trend in articles related to epidemiology published in occupational health journals. Occupational and environmental medicine 1996; 53: 433-438

17. Wichmann T, DeLong MR: Deep brain stimulation for neurologic and neuropsychiatric disorders. Neuron 2006; 52:197-204

18. Wisdom JP, Cavaleri MA, Onwuegbuzie AJ \& Green CA: Methodological reporting in qualitative, quantitative, and mixed methods health services research articles. Health services research 2012; 47:721-745

Correspondence:

Manik Sharma, MD

Department of CSA, DAV University Jalandhar

Jalandhar, India

E-mail:manik_sharma25@yahoo.com 